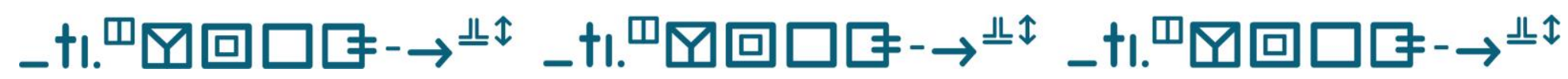

\section{Reflexões acerca do fazer ético e ativo dos intérpretes envolvendo línguas de sinais}

\section{Reflections on the ethical and active work of interpreters involving sign languages}

\footnotetext{
iD Eduardo Andrade Gomes

Universidade Federal de Juiz de Fora, Juiz de Fora, Minas Gerais, Brasil Universidade Federal de Minas Gerais, Belo Horizonte, Minas Gerais, Brasil edu.gomes06@gmail.com
}

(iD) 9

Oswaldo Vinícius Alves de Oliveira Rocha

Universidade Federal de São João del-Rei, São João del-Rei, Minas Gerais, Brasil

osw.rocha@hotmail.com

(iD) 9

William Silvino da Silva

Universidade Federal de Viçosa, Viçosa, Minas Gerais, Brasil wwilliamsilvino@hotmail.com

Resumo: Este artigo tende a ombrear-se a alguns estudos já existentes quanto aos dizeres éticos profissionais em consonância com a operacionalização prática dos intérpretes de/entre/para línguas de sinais frente às inúmeras demandas que o trabalho exige. Para tanto, são apresentadas três situações interpretativas: uma em contexto educacional superior, outra em ambiente jurídico em moldes de julgamento e, por fim, em atendimento clínico. Todas estão localizadas em um circunscrito factível a ocorrer com pessoas surdas, necessitando, portanto, de mediação. Buscamos ponderar, a partir das problematizações encontradas em cada um dos três eixos, como os intérpretes poderiam agir e se portar perante os dilemas vivenciados e, mesmo assim, 
(tentar) manter uma postura ética, reflexiva e atual que não comprometa o seu desempenho e que não prejudique ou não perturbe a relação estabelecida entre os agentes fonte e alvo.

Palavras-chave: Ética profissional. Intérpretes de/entre/para línguas de sinais. Reflexões.

Abstract: This article tends to be in line with some existing studies on professional ethics according to the practical operationalization of interpreters from/between/to sign languages in the face of the numerous demands that the work requires. For this reason, three interpretative situations are presented: one in a higher educational context, another in legal spaces in the form of judgment, and finally in clinical care. All are located in a possible place to occur with deaf people, requiring, therefore mediation. We sought to consider from the problematizations found in each of the three axes, how interpreters could act and behave in the presence of the perplexity experienced and yet (try) to maintain a reflexive and current ethical posture that does not compromise their performance and does not harm or disturb the relationship established between source and target agents.

Keywords: Professional ethics. Interpreters from/between/to sign languages. Reflections.

Submetido em 18 de junho de 2020. Aceito em 04 de setembro de 2020. Publicado em 02 de junho de 2021. 


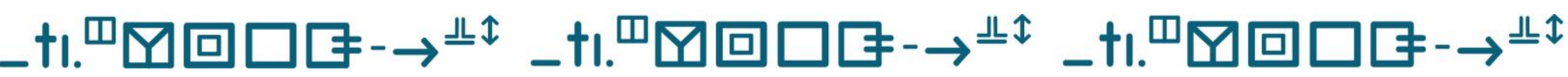

Reflexões acerca do fazer ético e ativo dos intérpretes de línguas de sinais Eduardo Andrade Gomes • Oswaldo Vinícius Alves de Oliveira Rocha • et al.

\section{Introdução}

A atuação dos intérpretes envolvendo línguas de sinais (ILS), seja em caráter intermodal (i.e., línguas de modalidades diferentes como a Libras e o português), seja intramodal gestual-visual (i.e., línguas de mesma modalidade, as de sinais), se pautou, durante muitos anos, em atividades de natureza voluntária e assistencialista, sendo desempenhadas, quase restritamente, por familiares, amigos e pessoas próximas aos surdos, tomados por múltiplos sentimentos e expectativas (QUADROS, 2004; RODRIGUES, 2019b). Dessas funções, a interpretação ganhou proporções numerosas pelo fato de lidar, em seu princípio básico, com a mediação linguístico-cultural de maneira interpessoal (i.e., face a face).

Entretanto, possivelmente, essas ações não eram desempenhadas do modo em que conhecemos hoje, em virtude da informalidade e, até mesmo, da indiferença que a sociedade mantinha com as pessoas surdas. Por isso, devido, inclusive, à lacuna de registros ao longo da narrativa histórica de pessoas que intermediavam discursos e relações entre o público, cuja língua ainda não era legitimada e prestigiada, e aqueles falantes da língua oficial do país, Quadros (2004) e Laguna (2015) os cita como intérpretes empíricos e Rodrigues (2019b) vai além ao identificá-los como precursores da interpretação. Essa marcação é importante, pois nos revela que toda a aquisição e o aprendizado em relação à língua de sinais e à cultura surda, por parte desses intérpretes, despontavam a partir do intenso e constante contato com a população surda, sendo entes ativos e parceiros nas lutas e nas causas aclamadas por esse grupo. 


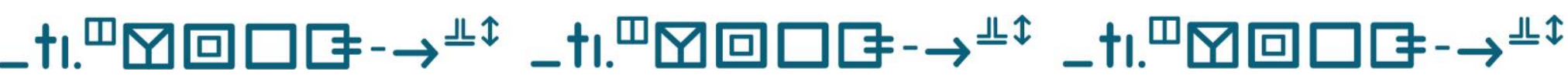

Reflexões acerca do fazer ético e ativo dos intérpretes de línguas de sinais Eduardo Andrade Gomes • Oswaldo Vinícius Alves de Oliveira Rocha • et al.

Ao avançar dos anos, após crescente e acentuada mobilização, os surdos foram conquistando seu espaço na sociedade e a língua de sinais alcançou seu estatuto linguístico e legal. Essas ações, apoiadas pelos ILS, também culminaram no desenvolvimento e no reconhecimento do trabalho e do profissionalismo desses agentes, se tornando evidente e nacionalmente considerado, por meio da regulamentação da Lei $n^{\circ}$ 12.319/2010. Esse passo foi essencial, uma vez que melhor qualificado, os profissionais poderão oferecer um serviço mais satisfatório aos solicitantes, tendendo a garantir, nessa concepção, os respectivos direitos linguísticos. Intuindo enriquecer essa qualificação profissional com exigência da formação superior em interpretação e tradução, dentre outras questões, o Projeto de Lei n 9.382/2017, após aprovação na Câmara dos Deputados no fim de 2020 e aguardando tramitação no Senado Federal, preconiza a revogação da legislação vigente da profissão dos ILS.

Assim, consoante à Rodrigues (2019b, p. 147), podemos reiterar que "a história dos intérpretes e tradutores de Português-Libras [Língua Brasileira de Sinais] se confunde com a história dos surdos e com a constituição e reconhecimento da Libras". O autor supracitado valida essa discussão ao sintetizar que

inicialmente, a atividade de interpretação era assumida e conduzida em uma perspectiva assistencialista e/ou de caridade, sem uma preocupação com a formação prévia ou profissional. A militância e o posicionamento político em prol dos surdos confundiam-se com a função dos intérpretes e a fluência em Libras apresentava-se como o 


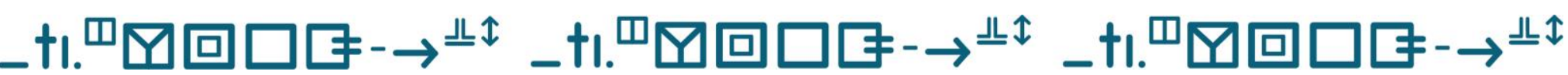

Reflexões acerca do fazer ético e ativo dos intérpretes de línguas de sinais Eduardo Andrade Gomes • Oswaldo Vinícius Alves de Oliveira Rocha • et al.

principal e, muitas vezes, único requisito à atuação (RODRIGUES, 2019b, p. 153).

Em tempos nos quais comemoramos a expansão, a formação e a profissionalização da carreira de intérpretes de/entre/para línguas de sinais e de tantas outras profissões no Brasil, o Governo Federal implementou, por meio do Decreto n 9.906/2019, o Programa Nacional de Incentivo ao Voluntariado, o Conselho do Programa Nacional de Incentivo ao Voluntariado, o Prêmio Nacional de Incentivo ao Voluntariado e o Selo de Acreditação do Programa Nacional de Incentivo ao Voluntariado. Por mais que as finalidades dessa ação sejam, no artigo 10, inciso I e II, "promover o voluntariado de forma articulada entre o Governo, as organizações da sociedade civil e o setor privado e incentivar o engajamento social e a participação cidadã em ações transformadoras da sociedade", e no artigo $20^{\circ}$ expressar que

a atividade voluntária não substitui o papel do Estado e que órgãos e entidades da administração pública e entidades privadas responsáveis por atividades voluntárias não poderão engajar voluntários em substituição a empregos e cargos formais ou como meio de evitar obrigações para com seus empregados e servidores (BRASIL, 2019).

parece-nos um tanto quanto inoportuna, pois além de remeter ao início da atuação dos ILS, abre margem, mesmo que inconscientemente, à sociedade creditar aos profissionais da interpretação, por exemplo, que sua atuação seja movida pelo voluntariado. Atitudes como essas podem ser encontradas em mídias sociais e em manifestações concretas oriundas do Tribunal Regional do Trabalho da $11^{\text {a }}$ 


\section{_t..}

Reflexões acerca do fazer ético e ativo dos intérpretes de línguas de sinais Eduardo Andrade Gomes • Oswaldo Vinícius Alves de Oliveira Rocha • et al.

região, da Secretaria de Justiça e Cidadania do Distrito Federal, do Instituto Federal de Educação Ciência e Tecnologia do Espírito Santo - campus Colatina1, e da Universidade Federal dos Vales do Jequitinhonha e Mucuri². É necessário que nos mantenhamos vigilantes, uma vez que a provável desestabilização de um movimento e de uma profissão não pode ser endossada na atual conjuntura em que a sociedade brasileira está minimamente estruturada.

Embora a legislação referente à profissão seja recente, 2010, conforme fora mencionado, a execução do trabalho já perpassa um longo período e, por isso, sempre foi necessário pensar em preceitos éticos em relação à procedência durante o trabalho. Segundo Quadros (2004) e Carneiro (2018), o código de ética é uma ferramenta capaz de orientar, nortear e balizar o profissional, no caso, o intérprete, em relação ao seu exercício laboral. Mais que elencar ou exigir diretrizes, ele é importante para tentar asseverar ou, pelo menos, respaldar os seus membros quanto à possibilidade de desenvolver seu trabalho de maneira consciente, efetiva, harmoniosa e respeitosa. Ratificando essas considerações, Carneiro (2018, p. 52) diz ser relevante "conhecer as regras do jogo, para segui-las ou subvertê-las conscientemente, saber em que ponto está a organização de sua classe profissional, saber da importância da regulamentação da profissão, [e] discutir princípios ético-profissionais ligados à sua área de atuação [...]". Afinal, para a autora, a intenção é que "os intérpretes prestem um bom serviço, deixem seus clientes satisfeitos e tenham seus direitos e deveres bem determinados, para que não haja reclamações entre as partes interessadas na contratação" (p. 39).

No Brasil, de acordo com Quadros (2004) e Carneiro (2018), o primeiro código de ética direcionado aos ILS é 


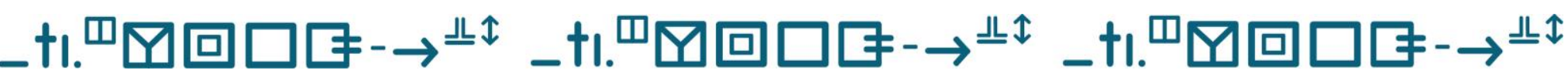

Reflexões acerca do fazer ético e ativo dos intérpretes de línguas de sinais Eduardo Andrade Gomes • Oswaldo Vinícius Alves de Oliveira Rocha • et al.

datado de 1992, aprovado em um encontro nacional da categoria, organizado pela Federação Nacional de Educação e Integração dos Surdos (FENEIS), por meio do seu Departamento nacional de Intérpretes, atualmente suprimido. Tal documento é fruto da tradução e da pouca adaptação do código de ética profusamente empregado nos Estados Unidos pelo Registry of Interpreters for the Deaf(RID - Registro de Intérpretes para Surdos, em português), cujas normativas se pautavam, explicitamente, em tomar decisões somente perante aos aspectos linguísticos das línguas envolvidas. Posteriormente, em território brasileiro, associações de ILS foram surgindo e criando os próprios códigos de ética, baseados em critérios do então documento da FENEIS e de outras associações de tradutores e intérpretes operantes entre línguas vocais-auditivas, como examinam Santos (2017) e Carneiro (2018). Após o nascimento da Federação Brasileira das Associações dos Profissionais Tradutores, Intérpretes e Guia-Intérpretes de Língua de Sinais (FEBRAPILS), em 2008, o primeiro código de (conduta e) ética da federação foi instituído em 2011 e reelaborado em 2014, cuja versão está em vigência.

Os profissionais da interpretação operam, a todo momento, variáveis fundamentais como (i) a sua própria subjetividade; (ii) as línguas em questão; (iii) os discursos enunciados; (iv) o público-fonte e o público-alvo; (v) as condições físicas e estruturais do ambiente de trabalho; (vi) a remuneração; entre tantas outras que podem impactar o processo e o produto textual. Por isso, diante de diversificados fatores reais, eles terão que tomar decisões e lançar estratégias que não podem estar em dissonância aos parâmetros éticos, para concluir com êxito a sua atividade. 


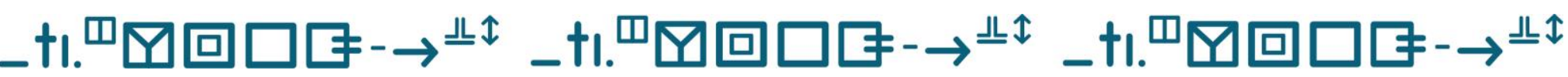

Reflexões acerca do fazer ético e ativo dos intérpretes de línguas de sinais Eduardo Andrade Gomes • Oswaldo Vinícius Alves de Oliveira Rocha • et al.

A fim de ilustrarmos essa temática, a seguir, apresentaremos uma discussão referente à ética, à moral, dialogadas ao trabalho dos ILS e às diversas demandas profissionais existentes, além do controle dispensado para contorná-las. Em seguida, abordaremos três cenários interpretativos, em que alguns fatores, inevitavelmente, mobilizarão atitudes éticas dos intérpretes ao desempenhar a sua atividade.

Para tanto, o presente estudo vem fazer coro aos apontamentos desenvolvidos por Nascimento (2014), VieiraMachado e Santana (2015), Cruz (2016), Santos (2017), Carneiro (2018) e Rosa e Monteiro (2018), caminhando no sentido de despertar nos ILS um espírito reflexivo em seu ofício de maneira a avaliar quais as melhores medidas poderiam ser adotadas frente ao vasto leque de circunstâncias e situações passíveis de ocorrer.

\section{1. Ética, tradução ${ }^{3}$ e interpretação: delineamentos factíveis}

Ética é um termo conhecido e utilizado na humanidade há muitos anos e que se fundamenta e entrelaça, entre outros, a concepções filosóficas, científicas, políticas, econômicas, sociais e humanas, conforme corroboram Valls (1993), Ferreira e Dias (2005) e Gesser (2011). Marchionni (2008 apud GESSER, 2011) ressalta que, etimologicamente, éthos preconiza o modo de ser intrínseco ao sujeito vinculado ao seu caráter, além dos princípios, valores, costumes e hábitos que podem se manifestar e se expressar nos atos concretos assumidos pelos indivíduos.

Nesse sentido, percebemos que, embora haja certa abstração em sua fecundação e maturação, por outro lado, a 


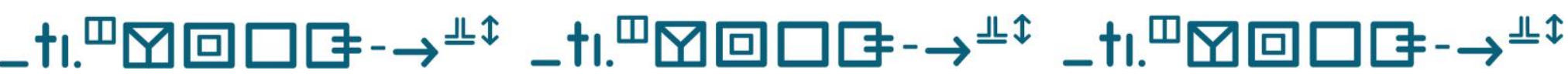

Reflexões acerca do fazer ético e ativo dos intérpretes de línguas de sinais Eduardo Andrade Gomes • Oswaldo Vinícius Alves de Oliveira Rocha • et al.

quanto à essência interdisciplinar desse campo, a sua real aplicabilidade e a expansão que o mesmo poderia obter. Mais tarde, a editora Saint Jerome Publishing 5 , especializada em publicações no âmbito dos Estudos da Tradução e da Interculturalidade, avolumou as temáticas para vinte e sete categorias ou subáreas, demonstrando, novamente, a sua consolidação como ramo de estudos e de pesquisas. Todavia, por mais que se tenha expandido as subdivisões e algumas temáticas sido expostas redundantemente, sobretudo no que tange à interpretação, a ética não surge de modo transparente, assim como em Williams e Chesterman (2002), permitindo que seja concebida como algo transcendente às outras categorias.

Carneiro (2018) desenvolve a mesma observação ao mencionar a proposição de Holmes (1972) ${ }^{6}$, que compreende os ET como eixos puro e aplicado, sendo que o aplicado engloba a formação do tradutor, as ferramentas de auxílio à tradução e a política/crítica da tradução. Concordamos com a autora ao arrazoar que a dimensão ética emerge em situações práticas e, por isso, essa discussão, apesar de não ser ostentada abertamente no mapa de James Holmes, poderia estar ligada, efetivamente, ao eixo aplicado.

Ainda que, em moldes acadêmicos e de pesquisa, a interpretação tenha sido abrigada, sem qualquer distinção, aos Estudos da Tradução, é essencial evidenciar que essa área possui contornos próprios e sólidos. Por isso, pesquisadores como o austríaco Franz Pöchhacker e o francês Daniel Gile defenderam e ecoaram a denominação específica para os Estudos da Interpretação (EI) como um campo muito mais independente do que afim aos ET. Tal medida está em consonância com todas as características e os procedimentos cognitivos, operacionais e situacionais em 


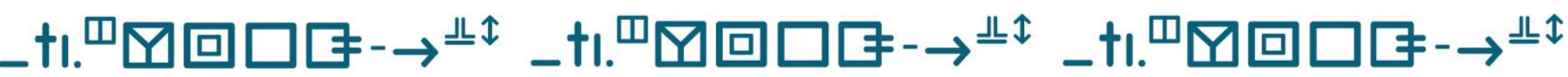

Reflexões acerca do fazer ético e ativo dos intérpretes de línguas de sinais Eduardo Andrade Gomes • Oswaldo Vinícius Alves de Oliveira Rocha • et al.

que a interpretação, o intérprete e o ato de interpretar estão imbuídos, destoando, portanto, da tradução (RODRIGUES, 2013). Devido ao exponencial crescimento da cristalização, da promoção e da veiculação de trabalhos e de pesquisas em torno das línguas de sinais e suas interfaces profissionais e acadêmicas teórico-metodológicas com a tradução e a interpretação, Rodrigues e Beer (2015) sugerem a denominação e a emergência do campo disciplinar Estudos da Tradução e da Interpretação de Línguas de Sinais (ETILS).

Em relação aos tradutores e aos intérpretes, independentemente de qual(is) seja(m) o(s) par(es) linguístico(s), alguns pontos nevrálgicos como (im)parcialidade, neutralidade, (in)fidelidade e confidencialidade ainda são concebidos como fio condutor de um código de ética (SANTOS, 2017). Em uma perspectiva mais ampla e despossuída de qualquer afiliação a alguma corrente teórica particular, os itens reportados poderiam ser encarados sob a invisibilidade imposta, sobretudo por lentes clássicas e rígidas, ao tradutor e ao intérprete. Essa invisibilidade não se enquadra necessariamente ao fator físico, mas acolhe todas as premissas de que o texto-fonte deva ser manipulado e transposto sem qualquer interferência por parte do tradutor ou do intérprete. Tal intervenção não se trata de alteração ao teor da mensagem, mas perpassa, entre outros, a leitura emocional, textual e cognitiva do profissional. Desse modo, Santos e Torres (2012) salientam a importância ao respeito pelas distinções culturais e linguísticas inerentes a todos os processos tradutórios e interpretativos, uma vez que os textos-fonte são materiais que permitem diversas compreensões e elucubrações (ALVES, 2021). 


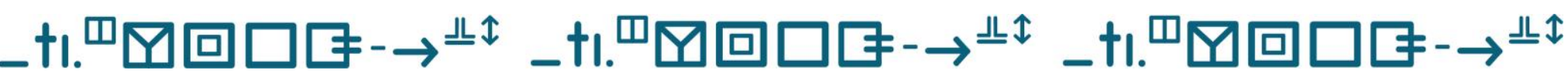

Reflexões acerca do fazer ético e ativo dos intérpretes de línguas de sinais Eduardo Andrade Gomes • Oswaldo Vinícius Alves de Oliveira Rocha • et al.

Nessa mesma linha de raciocínio, é válido acenar que discussões e reflexões entre os profissionais da área, o público-fonte e o público-alvo têm sido promovidas. Sendo assim, aponta-se um caminho para a (re)construção do sentido desses termos, reconhecendo as relações e as escolhas que cada um engendra em seu espaço, compreendendo que tais opções podem culminar em ganhos e em perdas.

Tratando-se principalmente da interpretação, assumir essa perspectiva que considere o dinamismo e a vitalidade das situações e dos agentes que circunscrevem os atos de reformulações linguístico-culturais é um aceno para novos horizontes, visto que os códigos de ética geralmente são normativos e prescritivos, conforme Nascimento (2014) argumenta.

No tocante à formação acadêmica inicial dos ILS em instituições federais de ensino, Rodrigues (2019a) realizou um levantamento do corpo das matrizes curriculares específicas à tradução e à interpretação. De acordo com os dados apresentados pelo autor, detectamos que a ética está explícita, em sua nomenclatura, enquanto disciplina e carga horária, em três universidades, a saber, Universidade Federal de Goiás, por "Ética na Tradução e Interpretação (64h)", Universidade Federal do Espírito Santo, com "Ética em Tradução e Interpretação (60h)" e Universidade Federal de São Carlos, em "Ética Profissional (30h)". Possivelmente, as demais instituições também tecem discussões a esse respeito, porém não apresentam uma disciplina singular para tal, como ocorre, por exemplo, na Universidade Federal de Santa Catarina, no curso em modalidade a distância - EaD em que esse tema é exaustivamente abordado em “Tradução e Interpretação de Língua de Sinais II (60h)". 


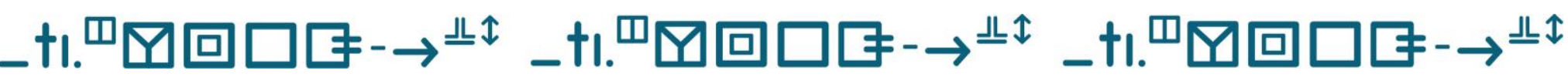

Reflexões acerca do fazer ético e ativo dos intérpretes de línguas de sinais Eduardo Andrade Gomes • Oswaldo Vinícius Alves de Oliveira Rocha • et al.

Concebemos a relevância em se ter um momento destinado exclusivamente para a reflexão dessa temática, mas acreditamos que esse assunto, ética, bem como seus conteúdos e desdobramentos, se faz necessário ao longo de todo o curso como um dos eixos transversais. Afinal, esse conceito e seus entendimentos irão emergir em várias atuações e situações práticas.

Os intérpretes de/entre/para línguas de sinais, assim como de outros pares linguísticos vocais-auditivos, são expostos cotidianamente a diversas situações e impressões externas e internas que podem influenciar o andamento e a conclusão das atividades. Para essa questão, Dean e Pollard (2001) a denominam como demandas. Defronte a essas imposições naturais do trabalho, o profissional necessitará mensurá-las ao tomar decisões, sendo essas conhecidas pelos autores como controle e, assim, reformulando, sobretudo para os ILS, a teoria da Demanda e do Controle. Chancelando essa afirmação,

O termo demanda refere-se aos requisitos de um trabalho, que podem incluir aspectos do ambiente, a tarefa real sendo executada e outros fatores que "agem" sobre o sujeito. O termo controle pauta-se ao grau de decisão em que o indivíduo tem para "agir de acordo com" as demandas apresentadas pelo trabalho (DEAN; POLLARD, 2001, p. 3) ${ }^{7}$.

Segundo os autores, as demandas externas e internas podem ser organizadas em quatro categorias, sendo a linguística, que detém aspectos relacionados estritamente às línguas em questão como, por exemplo, a fluência dos agentes, a prosódia (e.g., entonação, tom, volume da voz, postura e expressão corpóreo-facial), os léxicos em si e a estruturação sintática, semântica-pragmática e discursiva. 


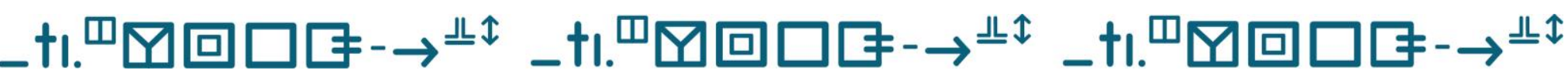

Reflexões acerca do fazer ético e ativo dos intérpretes de línguas de sinais Eduardo Andrade Gomes • Oswaldo Vinícius Alves de Oliveira Rocha • et al.

Outra demanda seria a ambiental, que engloba o espaço laboral, a (in)visibilidade física e sonora do profissional, a disposição espacial, a iluminação, a temperatura do ambiente, o uso de materiais de ordem sonora, visual e olfativa, entre outros.

A demanda interpessoal abarca as interações e as relações de poder entre o profissional e os demais membros do discurso, o controle na troca de turnos, quando há ILS de apoio, a consciência entre os envolvidos quanto às atribuições de cada um etc. Por fim, a demanda intrapessoal se atém a pontos de origem interna emocional e fisiológica, como o anonimato, a (in)segurança, o orgulho, a responsabilidade, os sintomas de nervosismo, a (in)visibilidade, o cansaço, a fome, o frio, entre outros.

Dean et al. (2020) ressaltam que essas duas demandas sobreditas (i.e., interpessoal e intrapessoal) podem sofrer influências entre si e, geralmente, os intérpretes não conseguem se expressar ou mesmo reconhecer, de maneira mais cristalina, as intrapessoais. Isso porque as demandas interpessoais se sobrepõem, e essas, por sua vez, imprimem, para os ILS, uma concepção de avaliação das atitudes dos seus interlocutores, implicando na percepção das demandas intrapessoais. Por isso, os autores recomendam que, ainda que haja uma interligação entre elas, o discernimento individual de ambas "permite que um intérprete reconheça a diferença entre esses dois tipos de demandas e trabalhe para encontrar respostas mais éticas e eficazes" (DEAN et al., 2020, p. 286).

Quando tais demandas surgem isolada ou concomitantemente, os ILS necessitam mobilizar, rapidamente, deliberações para enfrentá-las. Contudo, tanto as demandas quanto o controle podem despontar em uma 


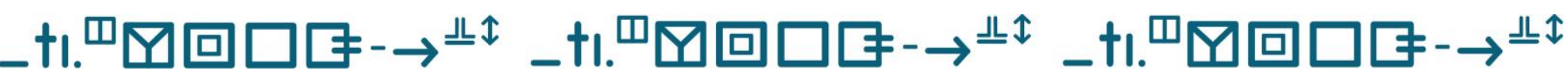

Reflexões acerca do fazer ético e ativo dos intérpretes de línguas de sinais Eduardo Andrade Gomes • Oswaldo Vinícius Alves de Oliveira Rocha • et al.

respeito ao direito de comunicação das pessoas, sendo uma das ações que podem fomentar a elaboração e a implementação de políticas linguísticas, como destacam Gonçalves Júnior et al. (2018). Frente a essa conjuntura de assimetria de poder, uma vez que haverá um membro que representa o Estado e outro que necessita dos serviços prestados por ele, Kalina (2015) frisa a importância de os intérpretes atuarem com vistas, sobretudo, a um maior grau de sensibilidade linguístico-cultural. Dessa forma, a interação estabelecida intentará atender os solicitantes em sua individualidade.

Apesar de não ser o nosso foco, Rodrigues e Santos (2018) defendem e exibem, por meio de quadros descritivos, que esses ambientes comunitários também podem abarcar atividades de natureza tradutória intermodal.

\section{Contornos éticos (possíveis) em situações interpretativas}

Nesta seção, apresentamos três situações interpretativas comunitárias hipotéticas, porém com alto potencial de ocorrência, uma vez que são baseadas na realidade vivida por ILS e clientes surdos.

Primeiramente, admitamos a atuação de um intérprete em modalidade simultânea de interpretação, com formação superior em Pedagogia, no âmbito educacional universitário, em aulas da componente curricular Física Eletromagnética do curso de Engenharia Civil. Na ocasião, o docente possui uma linguagem técnica robusta, bem como pouca articulação oral das palavras e baixo tom de voz. Por isso, não gosta que o ventilador seja acionado, o que gera um ambiente estrutural quente e abafado. Nesse caso, as demandas mais vigorosas 


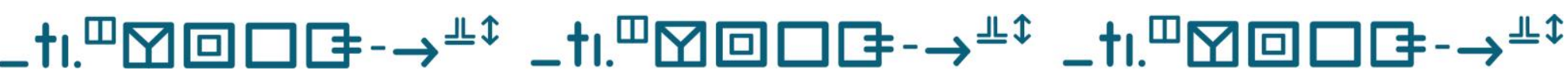

Reflexões acerca do fazer ético e ativo dos intérpretes de línguas de sinais Eduardo Andrade Gomes • Oswaldo Vinícius Alves de Oliveira Rocha • et al.

elencadas são a linguística e a ambiental, segundo Dean e Pollard (2001).

A linguística, por marcar a singularidade e a densidade lexical e terminológica como apontam Santiago e Lacerda (2016) no ensino superior com estudantes surdos, além da pouca prolação oral e volume de voz do professor, podendo comprometer, sobremaneira, a compreensão por parte dos ILS. A dificuldade em entender a mensagem do discursofonte tende a exigir do profissional a mobilização de esforços adicionais, o que pode abalar a sua organização em outras instâncias cognitivas como a memória e a produção linguística (GILE, 2009) ${ }^{9}$. Desta feita, seria interessante 0 profissional estar fisicamente mais próximo ao docente para conseguir ouvi-lo melhor e, caso tenha dúvida quanto aos termos, interpelar a aula e solicitar repetição e/ou maiores esclarecimentos. Outra postura respeitosa aconselhável seria, em momento apropriado após ou antes da aula, conversar com o docente e pedir o acesso ao material com antecedência ou o agendamento de um horário para sanar eventuais dúvidas, além de pontuar que o baixo tom de voz e a pouca articulação clara das palavras dificulta a compreensão, para então, sinalizar aos estudantes surdos.

Ademais, o exemplo dado expõe a demanda ambiental, espaço quente e abafado, também como um desafio para a concentração e a satisfação do intérprete para com o seu trabalho. Essa é uma questão delicada que talvez poderia ser conversada com o docente, visto que tal definição parte dele, mas o espaço da sala de aula com estudantes surdos também deveria ser regido, em um caráter de parceria, com o intérprete, como salienta Tesser (2015).

Uma nova situação a se pensar é quando um intérprete, com formação superior em andamento na área de 


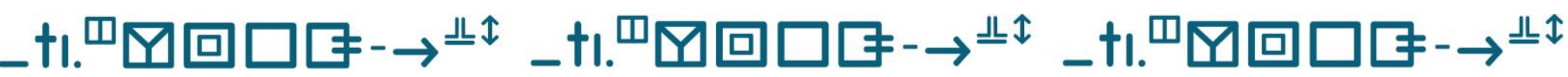

Reflexões acerca do fazer ético e ativo dos intérpretes de línguas de sinais Eduardo Andrade Gomes • Oswaldo Vinícius Alves de Oliveira Rocha • et al.

tecnologia, atuará em um contexto jurídico, que exige em grande parte do tempo a interpretação simultânea e possui termos técnicos e específicos. Esse profissional é convocado ad hoc para um julgamento em que o cidadão surdo, com pouco conhecimento, fluência e proficiência em Libras e em português, é a única testemunha ocular de um assassinato. Nesse espaço, há uma câmera/filmadora e um microfone direcionados a ele com o intuito de registrar todo o procedimento que poderá, conforme explanação do juiz durante a abertura do julgamento, ser auditado para fins de comprovação da fidelidade entre o texto-fonte e o texto-alvo. Para o andamento do processo, diante de tantas demandas, como a linguística, a intrapessoal e a interpessoal em que o intérprete está submetido, será preciso refletir sobre quais atitudes tomar. Provavelmente, haverá uma limitação comunicacional entre o intérprete e a testemunha. Em decorrência disso, seria importante deixar claro a todos quando houver uma dificuldade de compreensão das informações postas por ambas as partes.

O intérprete, em variados contextos, poderia buscar explorar mais gestos ou fenômenos linguísticos capazes de sustentar a criação e a efetivação de significação como ações construídas (i.e., incorporação de ações e atitudes como mecanismo referencial das entidades dos discursos) e classificadores (i.e., construções representativas, por meio de configurações de mãos, que permitem evidenciar e identificar os referentes dos enunciados), tentando ir ao encontro do ajustamento discursivo, reconhecendo a particularidade do sujeito surdo, como justificam Rosa e Monteiro (2018), ao abordarem, principalmente, o âmbito educacional. Além disso, dentro das possibilidades, orientar, mesmo durante a audiência, o uso de fotos, por exemplo, de pessoas 


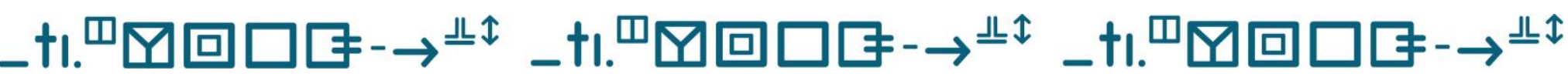

Reflexões acerca do fazer ético e ativo dos intérpretes de línguas de sinais Eduardo Andrade Gomes • Oswaldo Vinícius Alves de Oliveira Rocha • et al.

envolvidas na cena do crime, uma vez que a testemunha surda pode não reconhecer o nome das pessoas envolvidas. Ao verificar em imagens, poderá fornecer respostas contundentes e coerentes, evitando possíveis equívocos. Essa atitude considera e legitima uma das principais características essenciais e naturais das pessoas surdas enquanto seres dotados de humanidade, cultura e língua: a visualidade (CAMPELLO, 2008).

Mais um ponto que merece destaque é o eminente desconhecimento e despreparo do poder judiciário em lidar com questões que envolvam minorias linguísticas e profissionais para mediarem a comunicação. Assim, impõem alta carga de responsabilidade e, até mesmo, de intimidação aos ILS frente a uma questão tão séria como em um julgamento, conforme a redação de Santos (2016). A autora também introduz a adversidade posta pela terminologia jurídica aos intérpretes e, respaldada em Russell (2002), pontua a importância em se refletir quanto à aplicação da interpretação em modalidade consecutiva nesse contexto. Por fim, pondera o caminho profícuo e necessário a se traçar em função das melhorias de condições tanto para os atendidos quanto para os ILS nessa esfera de trabalho.

Ainda, outra situação contextual e extrema a ser refletida é a atividade de um intérprete, acionado via central de intérpretes da cidade, e que possui relações particulares estreitas com o requerente. Esse profissional dispõe de formação superior em História e interpretará em uma consulta médica na qual o paciente surdo entregará os exames realizados e receberá o diagnóstico de um tumor nos ossos. Uma das presumíveis demandas seria os termos técnicos que rondam o tratamento que precisará ser iniciado, bem como todos os novos procedimentos clínicos, 


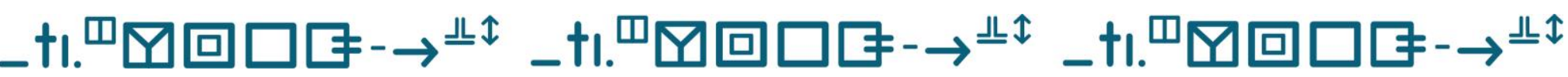

Reflexões acerca do fazer ético e ativo dos intérpretes de línguas de sinais Eduardo Andrade Gomes • Oswaldo Vinícius Alves de Oliveira Rocha • et al.

alimentares e medicamentosos em que o paciente se inserirá a partir de então.

Devido ao deslocamento de área, para melhor compreensão, o intérprete poderia solicitar ao médico que falasse mais pausadamente, dirimisse dúvidas quanto aos termos e optar pela interpretação consecutiva, modo mais utilizado em interpretações comunitárias, como Wadensjö (2009) afirma. Em meio a essas questões, está a importância de manter serenidade diante de uma notícia impactante tanto para o paciente surdo, quanto para o profissional que o conhece intimamente. Essas demandas linguísticas e interpessoais são alguns dos fatores que inquietam os intérpretes, conforme abordagem de Jesus (2017) em sua dissertação por meio de questionários, entrevistas e diário de campo.

Em todos os casos explanados, tentamos descortinar, mas não esgotar, algumas questões que os compõem e que poderiam ser admitidas, reconhecendo a natureza e a complexidade em torno dos contextos de atuação e dos agentes inseridos. Nesse sentido, as ações como (i) interromper o andamento da audiência para apresentar sugestões; (ii) solicitar esclarecimentos conceituais dos termos; e (iii) evidenciar a não compreensão do texto-fonte gerado pelo surdo, são algumas das ações éticas que, a princípio, poderiam não ser bem quistas e vistas, se ficássemos enrijecidos aos moldes tradicionais como os primeiros códigos de ética do RID e da FENEIS, por exemplo, alvitravam. Nesses documentos, era inaceitável qualquer aproximação ou elucubração, por conta dos ILS, durante o seu trabalho. A única premissa condizente seria a avaliação e a reflexão do ponto de vista linguístico, de maneira a manterse supostamente fiel e neutro aos oradores. 


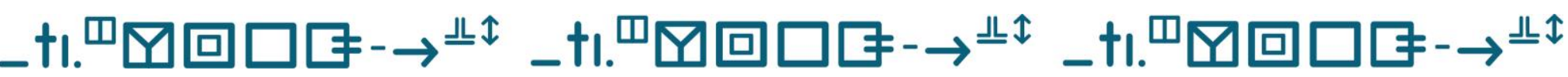

Reflexões acerca do fazer ético e ativo dos intérpretes de línguas de sinais Eduardo Andrade Gomes • Oswaldo Vinícius Alves de Oliveira Rocha • et al.

Todavia, Rosa e Monteiro (2018, p. 228) reforçam que "a ética não pode ser reduzida a algum código com leis gerais a serem aplicadas indistintamente, mas às possibilidades e necessidades do ato concreto no existir-evento". Diante desse engajamento e ponto de vista mais amplo e teleológico (i.e., baseado em objetivos e propósitos), Dean et al. (2020) discutem a noção de raciocínio ético aplicado em quaisquer situações. Tal raciocínio empenha-se a gerar no intérprete a criticidade e a noção do círculo interativo como um todo, em que o profissional, inevitavelmente, estará manuseando escolhas e tomadas de decisões de ordem linguístico-cultural, social, interpretativa etc, e as reavaliando constantemente. Esse quadro é alimentado pelo fato de existir, para cada resposta a uma demanda, uma possível ratificação ou retificação da mesma, até que o ato interpretativo em si se finde.

O atual código de conduta e ética profissional da FEBRAPILS (2014) preconiza como principais concepções a (i) confidencialidade; (ii) a competência tradutória; (iii) o respeito aos envolvidos na profissão; e (iv) o compromisso pelo desenvolvimento profissional. Embora, na perspectiva de Dean e Pollard (2001) o item (i) possa trazer certa limitação aos ILS em relação às demandas abordadas pelos autores, nas situações supracitadas, percebemos que as possíveis atitudes para contornar o cenário vivenciado poderiam estar alicerçadas, precipuamente, aos itens (ii) e (iv), ao reconhecer as necessidades impostas pelo acontecimento e, assim, articular todos os recursos possíveis na tentativa de melhor desempenhar seu papel. Esses recursos alinham-se à verificação das condições do ambiente, dos locutores e interlocutores envolvidos, do uso de materiais ou não durante a atividade, entre outros. 


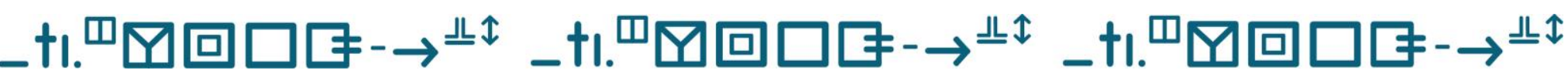

Reflexões acerca do fazer ético e ativo dos intérpretes de línguas de sinais Eduardo Andrade Gomes • Oswaldo Vinícius Alves de Oliveira Rocha • et al.

\section{Considerações finais}

Atuando voluntariamente, como nos primórdios, ou profissionalmente, os intérpretes envolvendo línguas de sinais sempre se deparam, em meio ao desempenho de sua função, com vivências, ora intensas, ora amenas, que evocam rápidas tomadas de decisão e de percepção dessas. Dean e Pollard (2001) intitulam esse mecanismo como teoria da Demanda e do Controle, que vem a ser uma vertente que tenciona a elucidar o que sempre vivemos: dimensionar as inúmeras sensações e situações impostas, sobretudo, em meio ao trabalho.

Assim, neste artigo, descrevemos três cenários interpretativos - educacional, jurídico e clínico objetivando destacar a diversidade de variáveis que os constituem, além de promover o pensamento de estratégias e soluções para as ocorrências resultantes de fatores linguísticos, ambientais, interpessoais e intrapessoais.

Nesse ínterim, concebemos que deva existir, conforme Dean et al. (2020) apontam, um raciocínio ético para que o profissional possa dosar, refletir e (re)avaliar a postura que irá assumir, mesmo que, a depender da situação, não seja tão simples.

Por isso, ressaltamos a importância da formação inicial e continuada para que esses profissionais compreendam a dimensão do ofício e se aprimorem. Essa ponderação, sem dúvidas, passará, além do viés técnico e estruturalista, por uma avaliação ética e moral, de maneira que eles consigam acomodar respeitosa e favoravelmente a definição da questão posta, tornando-se, como Vieira-Machado e Santana (2015) instilam, uma dicotomia entre o profissional enquanto indivíduo e a sua atuação abalizadamente prática. Pym (2012) 


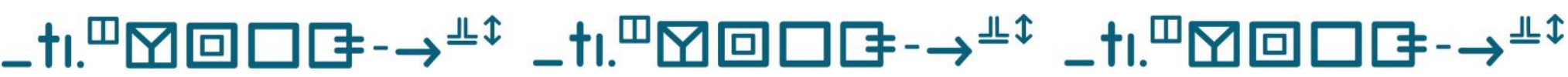

Reflexões acerca do fazer ético e ativo dos intérpretes de línguas de sinais Eduardo Andrade Gomes • Oswaldo Vinícius Alves de Oliveira Rocha • et al.

ainda notabiliza que as escolhas e os métodos tradutórios e interpretativos implicam em observações éticas para que sejam satisfatórias ao objetivo traçado em direcionar informações e conhecimentos entre as pessoas envolvidas.

\section{Referências}

ALVES, D. A. S. TRADUÇÃO E ÉTICA: SOBRE ÉTICA DA TRADUÇão COMO UMA PRÁTICA SOCIAL DE REFLEXÃO CONSCIENTE. REVISTA LINGUAGEM \& ENSINO, V. 24, N. 1, P. 1-12, 2021. DISPONÍVEL EM:< HTTPS://PERIODICOS.UFPEL.EDU.BR/OJS2/INDEX.PHP/RLE/ARTICLE/VIEW/ 20113>. ACESSO EM: 1 MAR 2021.

BRASIL. DECRETO 9.906. INSTITUI O PROGRAMA NACIONAL DE INCENTIVO AO VOLUNTARIADO, O CONSELHO DO PROGRAMA NACIONAL DE INCENTIVO AO VOLUNTARIADO, O PRÊMIO NACIONAL DE INCENTIVO AO VOLUNTARIADO E O SELO DE ACREDITAÇÃO DO PROGRAMA NACIONAL DE INCENTIVO AO VOLUNTARIADO. DIÁRIO OFICIAL DA UNIÃO. BRASÍLIA, O9 DE JULHO DE 2019. DISPONÍVEL EM:< HTTP://WWW.PLANALTO.GOV.BR/CCIVIL_03/_ATO20192022/2019/DECRETO/D9906.HTM>. ACESSO EM: 20 JUL 2019.

BRASIL. LEI 12.319. REGULAMENTA A PROFISSÃO DE TRADUTOR E INTÉRPRETE DE LÍNGUA BRASILEIRA DE SINAIS - LIBRAS. DIÁRIO OFICIAL DA UNIÃO. BRASÍLIA, 01 DE SETEMBRO DE 2010. DISPONÍVEL EM: $<$ HTTP://WWW.PLANALTO.GOV.BR/CCIVIL_03/_ATO20072010/2010/LEI/L12319.HTM>. ACESSO EM: 20 JUL 2019.

BRASIL. PROJeTO de LeI 9.382. DISPÕE SOBRE O EXERCíCIO PROFISSIONAL E CONDIÇÕES DE TRABALHO DO PROFISSIONAL TRADUTOR, GUIA-INTÉRPRETE E INTÉRPRETE DE LIBRAS, REVOGANDO A LEI № 12.319, DE $1^{\circ}$ DE SETEMBRO DE 2010. CÂMARA FEDeral dos Deputados. BRASÍLIA, 19 DE DEZEMBRO DE 2017. DISPONÍVEL EM: 


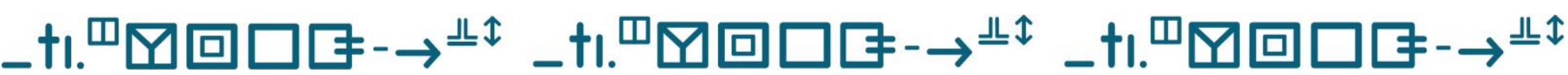

Reflexões acerca do fazer ético e ativo dos intérpretes de línguas de sinais Eduardo Andrade Gomes • Oswaldo Vinícius Alves de Oliveira Rocha • et al.

<HTTPS://WWW.CAMARA.LEG.BR/PROPOSICOESWEB/PROP_MOSTRARINT EGRA;JSESSIONID=NODE01XWWRMBPRM5A9DH84UVDXFVIK8878127.N ODEO?CODTEOR=1952632\&FILENAME=REDACAO+FINAL++PL+9382/2017>. ACESSO EM: 10 JAN 2021.

CAMPELLO, A. R. S. ASPECTOS DA VISUALIDADE NA EDUCAÇÃo DE SURDOS. 2008. 244 F. TESE DE DOUTORADO EM EDUCAÇÃO. UNIVERSIDADE FEDERAL DE SANTA CATARINA, FLORIANÓPOLIS, 2008. CARNEIRO, T. D. O PAPEL DOS CÓDIGOS DE ÉTICA E CONDUTA PROFISSIONAL NA FORMAÇÃO DO INTÉRPRETE DE LÍNGUAS ORAIS E DE SINAIS NO BRASIL. TRANSLATIO, N. 15, P. 33-56, 2018. DISPONível EM:

<HTTPS://SEER.UFRGS.BR/TRANSLATIO/ARTICLE/VIEW/80567/48557>. ACESSO EM: 20 JUN 2019.

CRUZ, R. M. H. CONFLITOS ÉTICOS NA ATUAÇÃO DO TRADUTOR INTÉRPRETE de LIBRAS. REVISTA VIRTUAL DE CULTURA SURDA, N. 17, P. 1-22, 2016.

DEAN, R.; POLLARD, R. Q. APPLICATION OF DEMAND-CONTROL THEORY TO SIGN LANGUAGE INTERPRETING: IMPLICATIONS FOR STRESS AND INTERPRETER TRAINING. JOURNAL OF DEAF STUDIES AND DEAF EDUCATION, P. 14, 2001.

DEAN, R. K; POLLARD JR, R. Q.; PENHA, L. C.; NASCIMENTO, V. RACIOCÍNIO ÉTICO BASEADO NO CONTEXTO DA INTERPRETAÇÃO: UMA PERSPECTIVA DO ESQUEMA DE CONTROLE DE DEMANDA. BELAS INFIÉIS, V. 9, N. 5, P. 269-299, 2020. DISPONÍVEL EM: $<$ HTTPS://PERIODICOS.UNB.BR/INDEX.PHP/BELASINFIEIS/ARTICLE/VIEW/2 8282/27878>. ACESSO EM: 15 JAN 2021.

FEBRAPILS, FEDERAÇÃO BRASILEIRA DAS ASSOCIAÇÕES DOS

PROFISSIONAIS TRADUTORES E INTÉRPRETES E GUIAINTÉRPRETES DE 


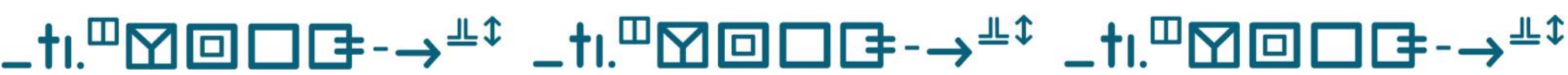

Reflexões acerca do fazer ético e ativo dos intérpretes de línguas de sinais Eduardo Andrade Gomes • Oswaldo Vinícius Alves de Oliveira Rocha • et al.

LÍNGUA DE SINAIS. Código de CONDUTA E ÉTICA. DISPONÍVEL EM: <HTTPS://DRIVE.GOOGLE.COM/FILE/D/OB7ZXCOYQ0QJMTUDTZ2XIZH LQQ1U/VIEW>, 2014.

FERREIRA, M. M.; DIAS, M. O. ÉTICA E PROFISSÃO RELACIONAMENTO INTERPESSOAL EM ENFERMAGEM, LOURES: LUSOCIÊNCIA. 2005.

GILE, D. BASIC CONCEPTS AND MODELS FOR INTERPRETER AND TRANSLATOR TRAINING. (REVISED EDITION). AMSTERDAM/PHILADELPHIA: JOHN BENJAMINS, 2009. GONÇALVES JÚNIOR, J.; DÓREA, Y. G. T.; KOGUT, M. K.; SOUZA, L. C. S. POLÍTICAS LINGUÍSTICAS E A LÍNGUA DE SINAIS BRASILEIRA. REVISTA SINALIZAR, V. 3, N. 1, P. 57-67, 2018. DISPONIVEL EM: <HTTPS://WWW.REVISTAS.UFG.BR/REVSINAL/ARTICLE/VIEW/51571/257 03>. ACESSO EM: 20 JUN 2019.

GESSER, A. TRADUÇÃo E INTERPRETAÇÃO DA LIBRAS II. TEXTO BASE DA DISCIPLINA DE TRADUÇÃO E INTERPRETAÇÃO DA LIBRAS II DO CURSO DE LETRAS - LIBRAS À DISTÂNCIA DA UNIVERSIDADE FEDERAL DE SANTA CATARINA. FLORIANÓPOLIS, P. 1-41, 2011. DISPONÍVEL EM: <HTTPS://WWW.LIBRAS.UFSC.BR/COLECAOLETRASLIBRAS/EIXOFORMACA OESPECIFICA/TRADUCAOEINTERPRETACAODALINGUADESINAIS/ASSETS/7 67/TEXTO_BASE_TIL_II_2008.PDF>. ACESSO EM: 10 ABR 2019.

HOLMES, J. S. THE NAME AND NATURE OF TRANSLATION STUDIES, 1972.

JESUS, R. B. “EI, AQUELE É O INTÉRPRETE DE LIBRAS?": ATUAÇÃO DE INTÉRPRETES DE LIBRAS NO CONTEXTO DA SAÚDE. 2017. 241 F. DISSERTAÇÃO DE MESTRADO EM ESTUDOS DA TRADUÇÃO. UNIVERSIDADE FEDERAL DE SANTA CATARINA, FLORIANÓPOLIS, 2017. DISPONÍVEL EM: < HTTPS://REPOSITORIO.UFSC.BR/BITSTREAM/HANDLE/123456789/1820 


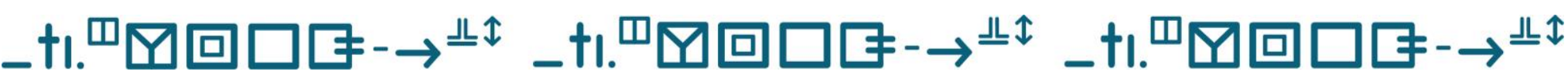

Reflexões acerca do fazer ético e ativo dos intérpretes de línguas de sinais Eduardo Andrade Gomes • Oswaldo Vinícius Alves de Oliveira Rocha • et al.

62/351452.PDF?SEQUENCE=1\&ISALLOWED=Y>. ACESSO EM: 10 ABR 2019.

KALINA, S. ETHICAL CHALLENGES IN DIFFERENT INTERPRETING SETTINGS. MONTI, V. 2, P. 63-86, 2015.

LAGUNA, M. C. V. MORALIDADE, IDONEIDADE E CONVIVÊNCIA: DISCURSOS SOBRE AS PRÁTICAS DOS REPETIDORES DE CLASSE DO INES NO PERÍODO DE 1855 A 1910 QUE INCIDEM NA ATUAÇÃO PROFESSIONAL DOS TRADUTORES-INTÉRPRETES DE LÍNGUA DE SINAIS DA ATUALIDADE. 2015. 157 F. DISSERTAÇÃO DE MESTRADO EM EDUCAÇÃO. UNIVERSIDADE FEDERAL DO RIO GRANDE DO SUL, PORTO ALEGRE, 2015. DISPONÍVEL EM: <HTTPS://LUME.UFRGS.BR/HANDLE/10183/128926>. ACESSO EM: 7 SET 2018.

NASCIMENTO, V. DIMENSÃO ERGO-DIALÓGICA DO TRABALHO DO TRADUTOR INTÉRPRETE DE LIBRAS/PORTUGUÊS: DRAMÁTICAS DO USO DE SI E DEBATE DE NORMAS NO ATO INTERPRETATIVO. REVISTA BRASILEIRA De LiNguística ApLICADA, V. 14, N. 4, P. 1121-1150, 2014. POINTURIER-POURNIN, S. L'INTERPRÉTATION EM LANGUE DES SIGNES FRANÇAISE: CONTRAENTES, TACTIQUES, EFFORTS, 2014, $460 \mathrm{~F}$. TESE DE DOUTORADO, UNIVERSITÉ PARIS 3, SORBONNE NOUVELLE, 2014.

PYM, A. TEORÍAS CONTEMPORÁNEAS DE LA TRADUCCIÓN. INTERCULTURAL STUDIES GROUP: TARRAGONA, P. 190, 2012.

QUADROS, R. M. O TRADUTOR E INTÉRPRETE DE LÍNGUA BRASILEIRA DE SinAis E LíngUA PORTUGUESA. BRASÍLIA: MEC, P. 89, 2004. RODRIGUES, C. H. A INTERPRETAÇÃo PARA A LÍNGUA DE SINAIS BRASILEIRA:EFEITOS DE MODALIDADE E PROCESSOS INFERENCIAIS. TESE 


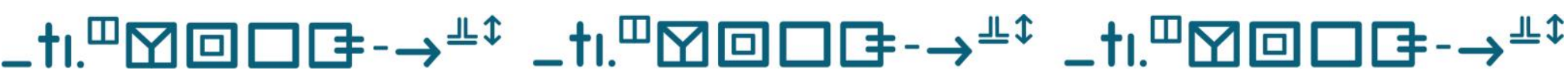

Reflexões acerca do fazer ético e ativo dos intérpretes de línguas de sinais Eduardo Andrade Gomes • Oswaldo Vinícius Alves de Oliveira Rocha • et al.

DE DOUTORADO EM LINGUístiCA APLICADA. 2013. 255 F.

UNIVERSIDADE FEDERAL DE MINAS GERAIS, BELO HORIZONTE, 2013.

RODRIGUES, C. H. O CORPO DE DISCIPLINAS DE TRADUÇÃO NA FORMAÇÃO DE TRADUTORES E INTÉRPRETES DE LÍNGUA DE SINAIS NO BRASIL: CONTEÚDOS, CARGA HORÁRIA E COMPETÊNCIAS. BELAS INFIÉIS, V. 8, N. 1, P. 145-162, 2019A. DISPONÍVEL EM: < HTTPS://PERIODICOS.UNB.BR/INDEX.PHP/BELASINFIEIS/ARTICLE/VIEW/12 775/20636>. ACESSO EM: 20 JAN 2020.

RODRIGUES, C. H. OS PROFISSIONAIS DA TRADUÇÃO E DA INTERPRETAÇÃO DE LÍNGUAS DE SINAIS NO BRASIL: PERCURSOS FORMATIVOS E PERFIL PROFISSIONAL. IN: MIRANDA, D. G.; FREITAS, L. (ORG.). EDUCAÇÃO PARA SURDOS: POSSIBILIDADES E DESAFIOS. BELO HORIZONTE: MAZZA EDIÇÕES, P. 147-164, 2019B.

RODRIGUES, C. H.; BEER, H. OS ESTUDOS DA TRADUÇÃO E DA INTERPRETAÇÃO DE LÍNGUAS DE SINAIS: NOVO CAMPO DISCIPLINAR emergente? Cadernos de TraduçÃo, v. 35, N. 2, P. 17-45, 2015. DISPONÍVEL EM: <HTTPS://PERIODICOS.UFSC.BR/INDEX.PHP/TRADUCAO/ARTICLE/VIEW/21 75-7968.2015V35NESP2P17/30707>. ACESSO EM: 12 JAN 2018.

RODRIGUES, C. H.; SANTOS, S. A. A INTERPRETAÇÃO E A TRADUÇÃO DE/PARA LÍNGUAS DE SINAIS: CONTEXTOS DE SERVIÇOS PÚBLICOS E SUAS DEMANDAS. TRADUÇÃo EM REVISTA, P. 1-29, 2018. DISPONÍVEL EM: <HTTPS://WWW.MAXWELL.VRAC.PUCRIO.BR/34535/34535.PDFXXVMI>. ACESSO EM: 30 SET 2019. ROSA, A. S.; MONTEIRO, M. I. B. ÉTICA NA INTERPRETAÇÃO DA LIBRAS-PORTUGUÊS NA SALA DE AULA: ALTERIDADE COMO FUNDAMENTO ÉTICO. TRANSLATIO, N. 15, P. 223-237, 2018. DisPONÍVEL EM: 


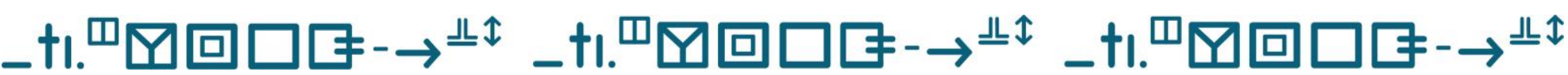

Reflexões acerca do fazer ético e ativo dos intérpretes de línguas de sinais Eduardo Andrade Gomes • Oswaldo Vinícius Alves de Oliveira Rocha • et al.

<HTTPS://SEER.UFRGS.BR/TRANSLATIO/ARTICLE/VIEW/80698/48555>. ACESSO EM: 20 JUN 2019.

RUSSELL, D. L. INTERPRETING IN LEGAL CONTEXTS: CONSECUTIVE AND SIMULTANEOUS INTERPRETATION. BURTONSVILLE, MD: LINSTOCK PRESS, P. 256, 2002.

SANTIAGO, V. A. A.; LACERDA, C. B. F. O INTÉRPRETE DE LIBRAS EDUCACIONAL: O PROCESSO DIALÓGICO E AS ESTRATÉGIAS DE MEDIAÇÃO NO CONTEXTO DA PÓS-GRADUAÇÃO. BELAS INFIÉIS, V. 5, N. 1, P. 165182, 2016. DISPONÍVEL EM: <HTTPS://PERIODICOS.UNB.BR/INDEX.PHP/BELASINFIEIS/ARTICLE/VIEW/1 1375/10012>. ACESSO EM: 11 JAN 2017.

SANTOS, G. B. F.; TORRES, M. C. REFLEXÕES SOBRE UMA ÉTICA NA TRADUÇÃO. BELAS INFIÉIS, V. 1, N. 1, P. 7-15, 2012. DISPONÍVEL EM: <HTTPS://PERIODICOS.UNB.BR/INDEX.PHP/BELASINFIEIS/ARTICLE/VIEW/1 1157/9810>. ACESSO EM: 20 JAN 2017.

SANTOS, S. A. QUESTÕES EMERGENTES SOBRE A INTERPRETAÇÃO DE LIBRAS-PORTUGUÊS NA ESFERA JURÍDICA. BELAS INFIÉIS, V. 5, N. 1, P. 117-129, 2016. DISPONÍVEL EM:

<HTTPS://PERIODICOS.UNB.BR/INDEX.PHP/BELASINFIEIS/ARTICLE/VIEW/1 1372/10009>. ACESSO EM: 11 JAN 2017.

SANTOS, W. ATUAÇÃO DE INTÉRPRETES DE LÍNGUA DE SINAIS: REVISITANDO OS CÓDIGOS DE ÉTICA. IN: ALBRES, N. A. (ORG.). LIBRAS E SUA TRADUÇÃO EM PESQUISA: INTERFACES, REFLEXÕES E METODOLOGIAS, P. 92-116, 2017.

TESSER, C. R. S. ATUAÇÃO dO INTÉRPRETE DE LIBRAS NA MEDIAÇÃo DA APRENDIZAGEM DE ALUNO SURDO NO ENSINO SUPERIOR: REFLEXÕES SOBRE O PROCESSO DE INTERPRETAÇÃO EDUCACIONAL. 2015. 


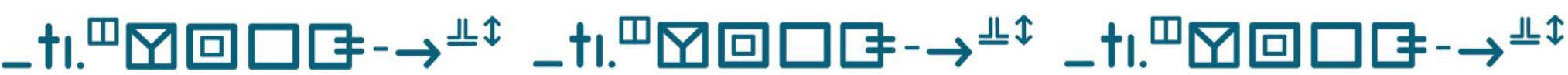

Reflexões acerca do fazer ético e ativo dos intérpretes de línguas de sinais Eduardo Andrade Gomes • Oswaldo Vinícius Alves de Oliveira Rocha • et al.

117 F. DISSERTAÇÃO DE MESTRAdO EM LINGUíSTICA. PONTIFÍCIA UNIVERSIDADE CATÓLICA DE SÃo PAULO, SÃO PAULO, 2015.

VALLS, A. L. M. O QUe É ÉTICA. SÃO PAULO: EDITORA BRASILIENSE, P. 80, 1993.

VIEIRA-MACHADO, L. M. D.; SANTANA, J. B. M. REFLEXÕES SOBRE

O TRABALHO DE TRADUÇÃO E INTERPRETAÇÃO EM LÍNGUAS DE SINAIS COMO PRÁTICA ÉTICA E POLÍTICA NO CUIDADO DE SI. CADERNOS DE

TRADUÇÃo, V. 35, N. 2, 149-173, P. 2015. DISPONÍVEL EM: < HTTPS://PERIODICOS.UFSC.BR/INDEX.PHP/TRADUCAO/ARTICLE/VIEW/217 57968.2015V35NESP2P149/30711>. ACESSO EM: 12 JAN 2018.

WADENSJÖ, C. COMMUNITY INTERPRETING. IN: BAKER, M.;

SALDANHA, G. (ORG.). ROUTLEDGE ENCYCLOPEDIA OF

TRANSLATION STUDIES. LONDON AND NEW YORK: ROUTLEDGE TAYLOR \& FRANCIS GROUP, 2ND EDITION, P. 43-48, 2009.

WILLIAMS, J.; CHESTERMAN, A. THE MAP: A BEGINNER'S GUIDE TO DOING RESEARCH IN TRANSLATION STUDIES. MANCHESTER: ST. JERONIME PUBLISHING, 2002.

\section{Notas}

\footnotetext{
${ }^{1}$ Maiores detalhes a respeito de atitudes empreendidas pelos três órgãos citados podem ser encontrados na página eletrônica da Federação Brasileira das Associações dos Profissionais tradutores, intérpretes e Guia-Intérpretes de Línguas de Sinais (FEBRAPILS): <https://drive.google.com/file/d/1WnYWA4FU3CHTwffDGIqMahUCawpQQOS6/view>

${ }^{2}$ Edital para a seleção de tradutores e intérpretes de Libras-Português voluntários: <https://portal.ufvjm.edu.br/noticias/2020/ufvjm-oferece-oportunidade-para-tradutores-e-interpretes-de-libras/edital-tilsp-voluntario-2020-1.pdf >
} 
Reflexões acerca do fazer ético e ativo dos intérpretes de línguas de sinais Eduardo Andrade Gomes • Oswaldo Vinícius Alves de Oliveira Rocha • et al.

${ }^{3}$ Esta seção comporta menções à tradução e ao tradutor, de forma a evidenciar que alguns aspectos são compartilhados com a interpretação e o intérprete. No entanto, as condições e os mecanismos cognitivos e procedimentais dessas tarefas são distintos, refletindo, inevitavelmente, em como os profissionais lidarão com elas. Neste artigo, por sua vez, as problematizações estão direcionadas à interpretação e aos intérpretes.

${ }^{4} \mathrm{O}$ esquema deste mapeamento pode ser deslumbrado em Williams e Chesterman (2002 apud RODRIGUES, 2013, p. 20).

${ }^{5} \mathrm{O}$ esquema da categorização desta editora pode ser figurado em Saint Jerome Publishing (2008 apud RODRIGUES, 2013, p. 22).

${ }^{6} \mathrm{O}$ esquema do mapa de Holmes pode ser visualizado em Pym e Turk (1998, p. 278 apud RODRIGUES, 2013, p. 19).

7 Tradução nossa de: "The term demand refers to the requirements of a job, which may include aspects of the environment, the actual task being performed, and other factors that "act upon" the individual. The term control refers to the degree to which the individual has the power to "act upon" the demands presented by the job, perhaps by making decisions".

${ }^{8}$ Tradução nossa de: "takes place in the public servisse sphere to facilitate communication between officials and lay people: at police departments, immigration departments, social welfare centres, medical and mental health offices, schools and other institutions".

${ }^{9}$ Essa questão reporta-se ao Modelo dos Esforços elaborado e revisitado pelo autor em operações de interpretação simultânea e consecutiva incluindo línguas de sinais. Para maiores esclarecimentos, consultar as publicações de Gile (2009) e de PointurierPournin (2014).

\section{Publisher}

UNIVERSIDADE FEDERAL DE GOIÁS. CURSOS DE LETRAS: LIBRAS E DE LETRAS: TRADUÇÃO E INTERPRETAÇÃO EM LIBRAS/PORTUGUÊS DA Faculdade de letras/ufg. Publicação no Portal de Periódicos UFG. AS IDEIAS EXPRESSADAS NESTE ARTIGO SÃO DE RESPONSABILIDADE DE SEUS AUTORES, NÃO REPRESENTANDO, NECESSARIAMENTE, A OPINIÃO DOS EDITORES OU DA UNIVERSIDADE. 